\title{
EGFR and HER2 Expression in Primary Ovarian High- Grade Serous Carcinoma and Their Prognostic Value
}

\author{
Mayada S. Farrag ${ }^{1}$, Ziad Emarah ${ }^{2}$, Waleed Elrefaie ${ }^{3}$, Nesrine S. Farrag ${ }^{4}$, \\ Mohamed T. Hafez ${ }^{5}$, Khaled Abdelwahab ${ }^{5}$
}

\begin{abstract}
${ }^{1}$ Department of Pathology, Faculty of Medicine, Port Said University, Port Said, Egypt; ${ }^{2}$ Medical Oncology Unit, Internal Medicine Department, Faculty of Medicine, Mansoura University, Mansoura, Egypt; ${ }^{3}$ Department of Obstetrics and Gynecology, Faculty of Medicine, Port Said University, Port Said, Egypt; 4 Department of Community Medicine and Public Health, Faculty of Medicine, Mansoura University, Mansoura, Egypt; ${ }^{5}$ Department of Surgical Oncology, Mansoura Oncology Center, Mansoura University, Mansoura, Egypt
\end{abstract}

\begin{abstract}
Background: Human epidermal growth factors receptors such as EGFR and HER2 play an important role in tumorigenesis and are used as therapeutic targets. Their role in aggressive primary ovarian high-grade serous carcinoma (HGSC) is controversial.

Aim: To study the expression of EGFR and HER2 in ovarian HGSC, to correlate their expression with other clinicopathological parameters and to study their prognostic value.

Methods: Imunohistochemical staining of EGFR, HER2 and Ki-67 was done for 54 ovarian HGSC specimens. According to the used scoring methods, the expression of EGFR and HER2 was classified as high or low.

Results: High expression of EGFR and HER2 was found in a minority of specimens; 39\% and 15\%, respectively. None of the studied clinicopathological parameters correlated significantly with the expression of EGFR and HER2, except for the carcinoembryonic antigen level which correlated positively with HER2 expression. Disease-free survival of patients did not differ significantly according to the level of expression of EGFR and HER2 ( $p=0.684$ and 0.186 , respectively). Similarly, overall survival did not differ significantly ( $p=0.911$ and 0.346 , respectively).

Conclusion: The results of this study suggest that the prognostic value of EGFR and HER2 in ovarian HGSC is questionable.

Keywords: EGFR, HER2, High-grade, Ovarian, Serous Carcinoma

Corresponding Author: Mayada S. Farrag, PhD; Department of Pathology, Faculty of Medicine, Port Said University, Port Said, Egypt; E-mail address: m.saad@med.psu.edu.eg

Submitted: 15-September-2020, Finally revised: 1-November-2020, Accepted: 2-November-2020, Published online: 28November-2020
\end{abstract}

\section{Introduction}

Ovarian cancer is the seventh most frequent cancer and the fifth cause of cancer deaths in women worldwide. The most common type is surface epithelial tumors. Unfortunately, it is mostly diagnosed at late stages ${ }^{1}$. Serous carcinoma is the most common and the most aggressive type of epithelial ovarian cancer ${ }^{2}$. Serous carcinomas are currently divided into two completely different subtypes, low grade serous carcinoma and highgrade serous carcinoma (HGSC), based on biological and histological morphologic features such as degree of nuclear atypia and number of mitoses ${ }^{3}$. Current therapy is based on few traditional predictive factors, such as tumor stage and postoperative tumor residual mass. Identification of new molecular markers could help in significant modification of treatment to improve clinical prognosis 4 .

The Erythroblastic oncogene B (ErbB) family of tyrosine kinase receptors plays an important role in development of several solid tumors. The abnormal activation of these receptors has been involved in different pathological processes such as 
cellular transformation ${ }^{5}$. Epidermal growth factor receptor (EGFR) is involved in many stages of cancer growth and development, such as tumor initiation, angiogenesis and also metastasis. In addition, it is included in many pathways as a proto-oncogene in several cancers like gastrointestinal and breast cancers. So, it is an attractive target for oncogenic therapy ${ }^{6}$.

Human epidermal growth receptor 2 (HER2) proto-oncogene is included in the genesis of many types of human cancers and is used as a therapeutic target. Although the correlation between HER2 expression and ovarian cancer has been studied before, the results are still controversial ${ }^{7}$. Therefore, in the present study we analyzed the expression of both EGFR \& HER2 in HGSC by immunohistochemistry, and results were correlated to prognosis.

\section{Methods}

\section{Design and patients}

This cross-sectional study was carried out on 54 specimens (archived paraffin blocks) of primary ovarian HGSC from patients who had been treated for ovarian cancer at the Oncology Centre, Mansoura University, Mansoura, Egypt in the period from 2012 to 2019. The following data were retrospectively-collected: age, tumor size (T), locoregional lymph node metastases $(\mathrm{N})$, distant metastases (M), International Federation of Gynecology and Obstetrics (FIGO) staging system, ascites, recurrence, residual tumor, carcinoembryonic antigen (CEA) level, Cancer Antigen 125 (CA125) level, peritoneal deposits and clinical outcomes (disease-free survival [DFS] and overall survival [OS]).

All patients received the same combination chemotherapy protocol (paclitaxel and carboplatin) either as neoadjuvant or adjuvant therapy.

\section{Immunohistochemical staining}

Sections were cut from paraffin-embedded tissue blocks at $4 \mu \mathrm{m}$, deparaffinized with xylene, then rehydrated with graded alcohols. The $1^{\text {ry }}$ antibodies included antibodies for EGFR (mouse monoclonal, Clone 111.6, Neo Markers, USA), HER2 (mouse monoclonal, clone CB-11, cell marque, USA) and Ki-67 (rabbit PAb, clone MIB-1, Neo Markers, USA). We used all of the antibodies according to manufacturer instructions.

We prepared appropriate positive controls (skin for EGFR and HER2 enriched tumor for HER2) and negative controls simultaneously with test slides. We performed antigen retrieval with heat in target retrieval solution $\mathrm{pH} 6.0$ for HER2 and Ki-67 and enzyme digestion with $0.05 \%$ protease $\mathrm{K}$ for $30 \mathrm{~min}$ at $37^{\circ} \mathrm{C}$ for EGFR.

\section{Immunohistochemical Analysis}

We evaluated EGFR by a scoring system based on both intensity and percentage of stained cells. The intensity score ranged from 1 to 3 . The threshold for positive reactions was $5 \%$. The score of the percentage was 1 (5-25\% positive cells), 2 (26-50\% positive cells), 3 (51-75\% positive cells) and 4 (>75\% positive cells). Final staining score (FSS) was calculated by multiplying both intensity and percentage scores, ranging from 1 to 12 . For the statistical analysis, FSS was considered as low expression for scores 1-4, and high expression for scores 6-12 ${ }^{5}$.

HER2 expression was assessed using Ellis and Wolff recommendations. Tumors were considered negative (low expression) with a score of 0 , and +1 . Tumors were considered positive (high expression) with a score of +3 when strong complete membranous staining observed in at least $10 \%$ of tumor cells ${ }^{8}$. Cases with a score of $2+$ were excluded from our study as they require fluorescence in situ hybridization (FISH) assay to confirm HER2 amplification.

For Ki-67, greater than $25 \%$ of cells positive was considered high expression, and lower than 25\% was considered low expression ${ }^{9}$.

\section{Statistical analysis}

Continuous data was presented in the form of mean + standard deviation (SD) or median + range (min-max) according to the results of Shapiro-Wilk testing for normal distributions of data. Categorical data was presented in the form of frequencies and percentages. Statistical significance was tested by Welch's t-test, or Mann-Whitney U for continuous data (according to the presence or absence of normal distribution of data). Chi-square test or Fisher's exact test were performed for categorical data (according to the minimal expected values in the contingencies tables). Correlations of different tumor markers were calculated using Spearman's rho coefficient. Kaplan-Meier method was used to estimate OS and DFS. Overall survival was calculated from the time of diagnosis to death and DFS from the end of treatment to the first recurrence / metastases or death. Only patients who achieved complete remission $(n=38)$ were 
included in DFS analysis. Comparison of survival was done using Log Rank (Mantel-Cox) test. Significance level was set at 0.05 .

Data was analyzed using the Statistical Package for the Social Sciences (SPSS) software version 16 for Windows.

\section{Ethical considerations}

The study was approved by Research Ethics Committee of the Faculty of Medicine, Mansoura University, Mansoura, Egypt (Approval code number: R.20.07.957).

\section{Results}

\section{Clinicopathological characteristics}

Clinicopathological features of 54 cases of HGSC are summarized in Table (1).

Nearly $61 \%$ and $85 \%$ of patients showed low expression of EGFR and HER2, respectively, while nearly $60 \%$ of cases showed high expression of Ki67 (Figure1).

\section{Correlation between the expression of EGFR and HER2 and the studied clinicopathological variables}

Table 2 summarizes the relation between the expression of EGFR and HER2 and the clinicopathological characteristics of patients.

The only variable that associated significantly with EGFR expression was the administration of neoadjuvant therapy. Expression of EGFR was significantly higher among patients who did not receive neoadjuvant treatment. Higher expression of EGFR was observed in patients who had ascites, residuals disease, and recurrence; however, this was not significant. There was no significant association between EGFR expression and other markers. The only variable that correlated significantly with HER2 expression was CEA level which correlated positively with it. Lower expression of HER2 was observed in patients with ascites, residual disease, recurrence and peritoneal deposits; however, the correlation was not significant.

\section{Correlation between the expression of HER2 and EGFR expression and survival}

The median duration of follow up was 40 months (range: 3.2-91.4).

The median OS was not reached in patients with low and high EGFR expression and the difference between them was not significant $(p=0.911)$ (Figure 2a). The median OS of patients with high Her2 expression was 59 months $(95 \%$ CI was not reached) and of those with low expression was not reached and the difference was not significant ( $p=$ 0.346) (Figure $2 b$ ).

There was no significant difference in DFS between patients with low EGFR expression and those with high expression (median in months $[95 \% \mathrm{CI}]=26.5$ [18.9-34] and 29 [0.5-58.2], respectively; $p=0.684$ ) (Figure $2 \mathrm{c}$ ). The DFS did not differ as well between patients with low Her2 expression and those with high expression (median in moths $[95 \% \mathrm{CI}]=29$ [17.3-40.7] and 22 [7.9-36.5], respectively; $p=0.186$ ) (Figure $2 \mathrm{~d}$ ).

Table 1: Clinicopathological characteristics of 54 patients with ovarian high-grade serous carcinoma

\begin{tabular}{|c|c|c|c|}
\hline \multicolumn{2}{|l|}{ Characteristic } & Mean (SD) & Total $n$ \\
\hline \multicolumn{2}{|l|}{ Age (years) } & $50(9.3)$ & 54 \\
\hline & & $n(\%)$ & \\
\hline \multirow[t]{2}{*}{$\mathrm{T}$} & T 1-2 & $10(25.6)$ & 39 \\
\hline & T 3-4 & $29(74.4)$ & \\
\hline \multirow[t]{2}{*}{$\mathbf{N}$} & $\mathrm{N} 0$ & $15(53.6)$ & 28 \\
\hline & N 1 & $13(46.4)$ & \\
\hline \multirow[t]{2}{*}{$\mathbf{M}$} & M 0 & $31(86.1)$ & 36 \\
\hline & M 1 & $5(13.9)$ & \\
\hline \multirow[t]{2}{*}{ FIGO Stage } & $1 / 2$ & $8(21.1)$ & 38 \\
\hline & $3 / 4$ & $30(78.9)$ & \\
\hline \multirow[t]{2}{*}{ Ascites } & No & $17(39.5)$ & 43 \\
\hline & Yes & $26(60.5)$ & \\
\hline \multirow[t]{2}{*}{ Residual tumor } & No & $22(56.4)$ & 39 \\
\hline & Yes & $17(43.6)$ & \\
\hline \multirow[t]{2}{*}{ Recurrence } & No & $17(44.7)$ & 38 \\
\hline & Yes & $21(55.3)$ & \\
\hline \multirow[t]{2}{*}{ Metastases } & No & $17(43.6)$ & 39 \\
\hline & Yes & $22(56.4)$ & \\
\hline \multirow{2}{*}{$\begin{array}{l}\text { Peritoneal } \\
\text { deposits }\end{array}$} & No & $9(20)$ & 45 \\
\hline & Yes & $36(80)$ & \\
\hline \multirow{2}{*}{$\begin{array}{l}\text { Neoadjuvant } \\
\text { therapy }\end{array}$} & No & $28(80)$ & 35 \\
\hline & Yes & $7(20)$ & \\
\hline \multirow{2}{*}{$\begin{array}{l}\text { Last known } \\
\text { status }\end{array}$} & Alive & $42(82.3)$ & 51 \\
\hline & Dead & $9(17.6)$ & \\
\hline \multirow{2}{*}{$\begin{array}{l}\text { EGFR } \\
\text { expression }\end{array}$} & Low & $33(61.1)$ & 54 \\
\hline & High & $21(38.9)$ & \\
\hline \multirow{2}{*}{$\begin{array}{l}\text { HER2 } \\
\text { expression }\end{array}$} & Low & $46(85.2)$ & 54 \\
\hline & High & $8(14.8)$ & \\
\hline \multirow[t]{3}{*}{ Ki-67 } & Low & $21(39.6)$ & 53 \\
\hline & High & $32(60.4)$ & \\
\hline & & $\begin{array}{l}\text { Median } \\
\text { (range) }\end{array}$ & \\
\hline CEA & & $2.2(0.1-64)$ & 15 \\
\hline CA-125 & & $220(7-1268)$ & 28 \\
\hline
\end{tabular}

CEA: Carcinoembryonic antigen, FIGO: International Federation of Gynecology and Obstetrics, SD: Standard deviation. 

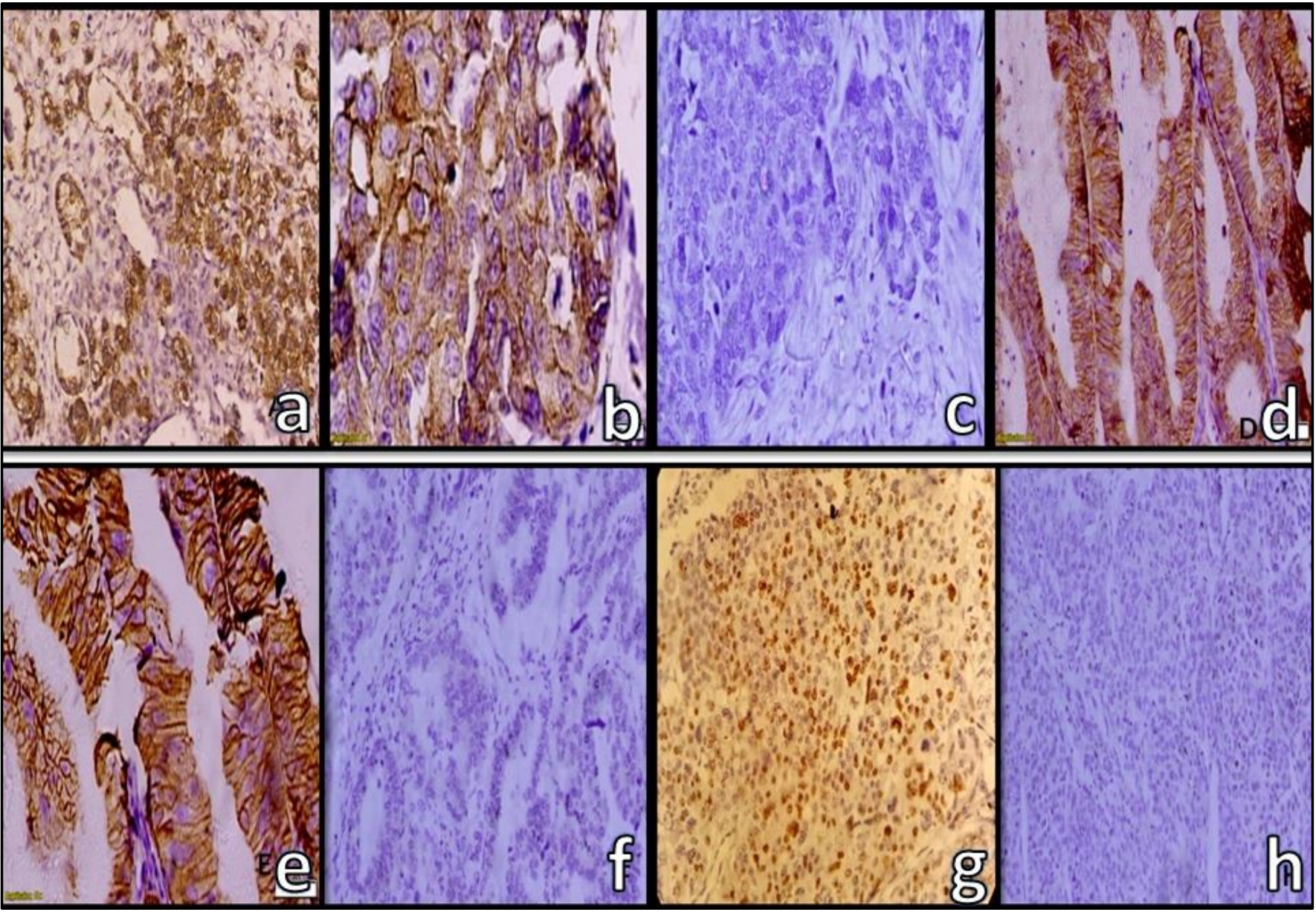

Figure 1: Immunohistochemical staining of EGFR, HER2\& Ki67 in different cases of ovarian high-grade serous carcinoma (a) High EGFR expression showing positive cytoplasmic staining, FSS=12, X100; b) High EGFR expression showing positive membranous staining, $\mathrm{FSS}=12$, X400; c) Low EGFR expression showing negative staining, FSS $=0$, X200; d) High HER2 expression showing positive complete membranous staining in $>10 \%$ of tumor cells, score +3 , X100; e) High HER2 expression showing positive complete membranous staining in $>10 \%$ of tumor cells, score +3 , X200; f) Low HER2 expression showing negative staining, x100); g) High K-i67 proliferative marker, X200, h) Low Ki-67 proliferative marker, X200], FSS: Final staining score

\section{Discussion}

In the present study, both HER2 and EGFR expressions were analyzed using immunohistochemistry in cases with primary ovarian HGSC. EGFR and its family members play a variety of roles in the growth of different cell types ${ }^{10}$. Also, EGFR is involved in various stages of cancer cells proliferation, being an attractive target for cancer therapy ${ }^{6}$. It could also affect the tumor response to targeted to targeted therapeutic agents which bind to the receptor/ligand ErbB system ${ }^{5}$.

Normally, the ovarian epithelium expresses EGFR poorly ${ }^{6}$. Both the metaplastic ovarian epithelium and tubal epithelium variably express EGFR ${ }^{2}$.
In ovarian carcinomas, expression of EGFR differs greatly among different studies ${ }^{11,12}$. Nielsen et al ${ }^{13}$ found that the EGFR positivity in both ovarian borderline tumors and carcinomas was significantly higher than in normal ovarian tissue and benign tumors. But Brustmann ${ }^{14}$ and Fujiwara et $\mathrm{al}^{15}$ found lack of positivity in borderline tumors when compared to serous carcinomas. EGFR expression is more common in ovarian serous carcinomas than borderline tumors and a number of studies found that it is significantly higher in HGSC 2, 5, 10, 14,16 . In ovarian carcinoma cells, suppression of EGFR may result in regression of aneuploidy and genomic imbalances restoring a more normal phenotype ${ }^{2}$.

The significance of EGFR expression in HGSC is still a matter of controversy ${ }^{14}$. So, we studied its expression in these cases which respond poorly to 
the usual combination chemotherapy protocols and correlated its expression with different clinicopathological parameters and with patient prognosis. We found high EGFR expression in $38.9 \%$ of HGSC patients. As regards the clinicopathological parameters such as tumor size, lymph node metastases, distant metastases, FIGO stage, ascites, recurrence, residual tumor, CA125 level, CEA level, Ki-67 proliferation marker and peritoneal deposits; we did not find a significant association with EGFR expression. Regarding correlation to patients' age, it differs from Lassus et al ${ }^{2}$ who found higher expression in patients of older age.

Our results regarding correlation of EGFR expression and tumor stage is in agreement with Brustmann ${ }^{14}$ and Mehner et al ${ }^{17}$ who found no significant association with tumor stage. On the contrary, Cîrstea et al 5 said that EGFR overexpression was more in advanced stage carcinomas but their study has limitations. We also did not find a significant association between EGFR overexpression and residual tumor or high cell proliferation index.

On the contrary, an association between increased EGFR expression and large residual tumor and high cell proliferation index was previously reported ${ }^{2,10}$. These discrepancies may be attributed to the different antibody clones used and staining methodology.

In the current study, the expression of EGFR was significantly higher among patients who did not receive taxol-carboplatin neoadjuvant treatment than those who received. This association was not explored in previous studies.

Table 2: The relation between EGFR and HER2 expression and patients' clinicopathological characteristics

\begin{tabular}{|c|c|c|c|c|c|c|c|}
\hline \multirow[t]{2}{*}{ Characteristic } & & \multicolumn{2}{|c|}{ EGFR expression } & \multirow[t]{2}{*}{$p$ value } & \multicolumn{2}{|c|}{ HER2 Expression } & \multirow[t]{2}{*}{$p$ value } \\
\hline & & Low & High & & Low & High & \\
\hline & & \multicolumn{2}{|c|}{ Mean (SD) } & & \multicolumn{2}{|c|}{ Mean (SD) } & \\
\hline \multirow[t]{2}{*}{ Age (years) } & & $52(8.7)$ & $48(9.9)$ & $0.270^{a}$ & $50.5(9.7)$ & $51.6(6.1)$ & $0.706^{a}$ \\
\hline & & \multicolumn{2}{|c|}{$n(\%)$} & & \multicolumn{2}{|c|}{$n(\%)$} & \\
\hline \multirow[t]{2}{*}{$\mathrm{T}$} & $\mathrm{T} 1-2$ & $6(60)$ & $4(40)$ & \multirow[t]{2}{*}{$1^{b}$} & $7(70)$ & $3(30)$ & \multirow[t]{2}{*}{$0.096^{b}$} \\
\hline & T 3-4 & $19(65.5)$ & $10(34.5)$ & & $27(93.1)$ & $2(6.9)$ & \\
\hline \multirow[t]{2}{*}{$\mathbf{N}$} & N0 & $10(66.7)$ & $5(33.3)$ & \multirow[t]{2}{*}{$1^{b}$} & $13(86.7)$ & $2(13.3)$ & \multirow[t]{2}{*}{$1^{\mathrm{b}}$} \\
\hline & N1 & $8(61.5)$ & $5(8.5)$ & & $11(84.6)$ & $2(15.4)$ & \\
\hline \multirow[t]{2}{*}{$\mathbf{M}$} & M0 & $19(61.3)$ & $12(38.7)$ & \multirow[t]{2}{*}{$1^{b}$} & $28(90.3)$ & $3(9.7)$ & \multirow[t]{2}{*}{$0.466^{b}$} \\
\hline & M1 & $3(60)$ & $2(40)$ & & $4(80)$ & $1(20)$ & \\
\hline \multirow[t]{2}{*}{ FIGO stage } & $1-2$ & $4(50)$ & $4(50)$ & \multirow[t]{2}{*}{$0.687^{b}$} & $6(75)$ & $2(25)$ & \multirow[t]{2}{*}{$0.279^{b}$} \\
\hline & $3-4$ & $19(63.3)$ & $11(36.7)$ & & $27(90)$ & $3(10)$ & \\
\hline \multirow[t]{2}{*}{ Ascites } & No & $11(64.7)$ & $6(35.3)$ & \multirow[t]{2}{*}{$0.834^{\mathrm{b}}$} & $14(82.4)$ & $3(17.6)$ & \multirow[t]{2}{*}{$0.666^{b}$} \\
\hline & Yes & $16(61.5)$ & $10(38.5)$ & & $23(88.5)$ & $3(11.5)$ & \\
\hline \multirow{2}{*}{$\begin{array}{l}\text { Residual } \\
\text { tumor }\end{array}$} & No & $16(72.7)$ & $6(27.3)$ & \multirow[t]{2}{*}{$0.201^{\mathrm{b}}$} & 17(77.3) & $5(22.7)$ & \multirow[t]{2}{*}{$0.056^{\mathrm{b}}$} \\
\hline & Yes & $9(52.9)$ & $8(47.1)$ & & 17 & 0 & \\
\hline \multirow[t]{2}{*}{ Recurrence } & No & $11(64.7)$ & $6(35.3)$ & $0.635^{b}$ & $14(82.4)$ & $3(17.6)$ & $1^{\mathrm{b}}$ \\
\hline & Yes & $12(57.1)$ & 9 (42.9) & & $18(85.7)$ & $3(14.3)$ & \\
\hline Metastasis & No & $10(58.8)$ & $7(41.2)$ & $0.759^{b}$ & $16(94.1)$ & $1(5.9)$ & $0.206^{b}$ \\
\hline & Yes & $14(63.6)$ & $8(36.4)$ & & $17(77.3)$ & $5(22.7)$ & \\
\hline Peritoneal & No & $6(66.7)$ & $3(33.3)$ & $0.721^{b}$ & $7(77.8)$ & $2(22.2)$ & $0.614^{b}$ \\
\hline deposits & Yes & $21(58.3)$ & $15(41.7)$ & & $31(86.1)$ & 5 (13.9) & \\
\hline Neoadjuvant & No & $4(14.3)$ & $24(85.7)$ & $0.001^{b}$ & $24(85.7)$ & $4(14.3)$ & $1^{b}$ \\
\hline therapy & Yes & $6(85.7)$ & $1(14.3)$ & & $6(85.7)$ & $1(14.3)$ & \\
\hline HER2 & Low & $28(60.9)$ & $18(39.1)$ & 1 & --- & --- & $\begin{array}{l}-- \\
\end{array}$ \\
\hline & High & $5(62.5)$ & $3(37.5)$ & & --- & --- & \\
\hline Ki-67 & Low & $14(66.7)$ & $7(33.3)$ & $0.448^{b}$ & $20(95.2)$ & $1(4.8)$ & $0.126^{b}$ \\
\hline & High & $18(56.2)$ & $14(43.8)$ & & $25(78.1)$ & $7(21.9)$ & \\
\hline & & Med & (range) & & Speal & man's rho & \\
\hline CEA & & $2.2(0.3-13)$ & $0.35(0.1-0.6)$ & $0.759^{c}$ & & 0.536 & $0.039^{d}$ \\
\hline CA 125 & & $\begin{array}{l}212(104- \\
1268)\end{array}$ & $\begin{array}{l}324.5 \text { (201- } \\
448)\end{array}$ & $0.555^{c}$ & & 0.034 & $0.865^{d}$ \\
\hline
\end{tabular}

CEA: Carcinoembryonic antigen, FIGO: International Federation of Gynecology and Obstetrics, SD: Standard deviation. 

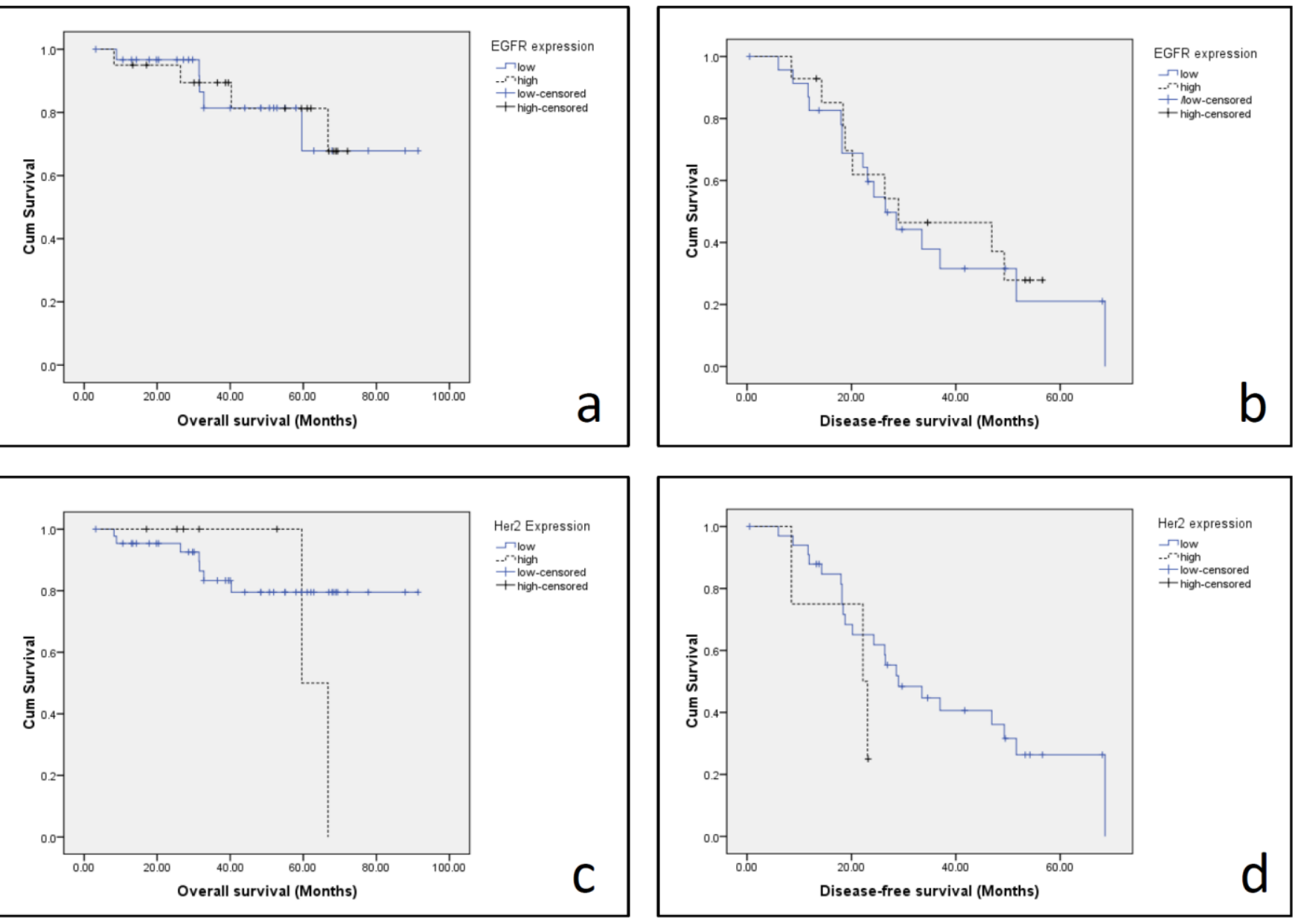

Figure 2: Kaplan-Meier survival curves [a) Overall survival according to EGFR expression, b) Overall survival according to HER2 expression, c) Disease-free survival according to EGFR expression and d) Disease-free survival according to HER2 expression]

The role of HER2 in ovarian cancer has been studied previously in some studies, but the results remain controversial ${ }^{7}$. Wang et al ${ }^{18}$ demonstrated the positivity for HER2/neu in benign and borderline ovarian tumors. Also, Other studies found positivity for HER2/neu in many borderline tumors without being a marker of malignancy ${ }^{5,16}$.

As regards HER2 expression in HGSC, we found high expression of HER2 in only $14.8 \%$ of patients. The percentage of patients with HER2-positive ovarian cancer differed considerably in previous studies and varied from $8 \%$ to $66 \% 5,8,19-21$. NofechMozes et al ${ }^{22}$ did not identify any cases of malignant serous ovarian tumor with HER2 overexpression. Other studies reported a small number of tumors with immunostaining for HER2 indicating that few patients with ovarian cancer have tumors that would benefit from specific targeted therapy ${ }^{23}$.

We did not find a significant association between HER2 expression in ovarian HGSC and any of the studied clinicopathological variables except the level of CEA. Contrary to our results, in the study done by Cîrstea et al ${ }^{5}$, a significantly higher values of HER2 expression was observed in advanced stage tumors; but this result was statistically unclear.

The survival analysis showed that OS and DFS did not differ significantly according to the expression of EGFR in ovarian HGSC. The lack of EGFR prognostic impact on the survival of ovarian HGSC found in this study is supported by a large study conducted by Mehner et al ${ }^{17}$ on different types of ovarian cancer. Our findings are in general accordance with what has been found in previous studies dealing with overall populations and EGFR staining as a prognostic marker in ovarian cancer. They found no significant association with survival 17,23 .

Other studies suggested that EGFR is associated with poor prognosis ${ }^{6,10,12,14,24}$. Also, Lassus et al ${ }^{2}$ found that EGFR expression was linked to shorter DFS. But none of these studies focused on HGSC. They studied EGFR expression in ovarian cancers 
in general. Many studies using smaller patient cohorts or limited patient subsets have recorded these significant associations. The study done by Cîrstea et $\mathrm{al}^{5}$ focused on EGFR expression in HGSC. They reported an association between high EGFR expression and poor prognosis and shorter survival times. But the numbers used for calculating significance were not clear.

As regards HER2, our survival analysis showed that OS and DFS times in patients with high HER2 expression were less than those in patients with low expression. However, these differences were not statistically significant. Some previous reports suggested that high expression of HER2 is a poor prognostic factor in ovarian cancer while others showed that HER2 overexpression had no impact on survival ${ }^{7}$. Luo et al ${ }^{7}$ found that the expression of HER2 was negatively correlated with OS and DFS in ovarian cancer. Researchers of studies in which HER2 overexpression was associated with poor prognosis recommended it as a valuable target for targeted therapy agents ${ }^{25,}{ }^{26}$. However, none of these studies focused on HGSC. Luo et al ${ }^{7}$ concluded that the prognostic value of HER2 expression is noted especially in patients with unclassified ovarian cancer and Caucasian origin. The study done by Cîrstea et al ${ }^{5}$ investigated HER2 expression in ovarian HGSC. They reported the association of high HER2 expression with poor prognosis and shorter survival times.

This study has limitations. It was a single center study that included a small number of patients. In addition, the survival data was missing in a proportion of patients and those with survival data were followed up for a relatively short time. Consequently, survival analysis results should be interpreted cautiously.

\section{Conclusion}

The results of the current study suggest that the prognostic value of EGFR and HER2 in ovarian HGSC is questionable. Subsequently, targeting these agents in the treatment of ovarian HGSC is not clearly suitable. Further adequately-powered studies with better selection and stratification of ovarian HGSC patients are needed to explore the benefit from HER2 and EGFR targeted therapy.

\section{Acknowledgment \\ None.}

\section{Authors' contribution}

Conception or design: MSF; Acquisition, analysis or interpretation of data: All authors; Drafting the manuscript: MSF; Revising the manuscript: ZE, WE, NSF, MTH, KA;
Approval of the manuscript version to be published: All authors; Agreement to be accountable for all aspects of the work: All authors.

\section{Conflict of interest}

The authors declare that they have no conflict of interest to disclose

\section{Data availability}

Deidentified individual participant data used to produce the results of this study are available from the corresponding author (MSF) upon request.

\section{Funding}

The authors did not receive funding for this study.

\section{Study registration}

ClinicalTrials.gov ID NCT04284631.

\section{References}

1. Rosso M, Majem B, Devis L, et al. E-cadherin: A determinant molecule associated with ovarian cancer progression, dissemination and aggressiveness. PLoS One. 2017; 12(9): e0184439.

2. Lassus H, Sihto $H$, Leminen $A$, et al. Gene amplification, mutation, and protein expression of EGFR and mutations of ERBB2 in serous ovarian carcinoma. J Mol Med (Berl). 2006; 84(8): 671-681.

3. Smolle E, Taucher V, Pichler M, Petru E, Lax S, Haybaeck J. Targeting signaling pathways in epithelial ovarian cancer. Int J Mol Sci. 2013; 14(5): 9536-9555.

4. Koensgen D, Freitag C, Klaman I, et al. Expression and localization of e-cadherin in epithelial ovarian cancer. Anticancer Res. 2010; 30(7): 2525-2530.

5. Cîrstea $\mathrm{AE}$, Stepan $\mathrm{AE}$, Mărgăritescu C, Zăvoi RE, Olimid DA, Simionescu CE. The immunoexpression of EGFR, HER2 and HER3 in malignant serous ovarian tumors. Rom J Morphol Embryol. 2017; 58(4): 1269-1273.

6. Cîrstea AE, Stepan AE, Zăvoi RE, Simionescu CE. EGFR immunoexpression in malignant serous and mucinous ovarian tumors. Curr Heal Sci J. 2018; 44(2): 129-134.

7. Luo H, Xu X, Ye M, Sheng B, Zhu X. The prognostic value of HER2 in ovarian cancer: A meta-analysis of observational studies. PLoS One. 2018; 13(1): e191972.

8. Tuefferd M, Couturier J, Penault-Llorca F, et al. HER2 status in ovarian carcinomas: A multicenter GINECO study of 320 patients. PLoS One. 2007; 2(11): e1138.

9. Wang K, Li D, Sun L. High levels of EGFR expression in tumor stroma are associated with aggressive clinical features in epithelial ovarian cancer. Onco Targets Ther. 2016; 9: 377-386.

10. Siwak DR, Carey M, Hennessy BT, et al. Targeting the epidermal growth factor receptor in epithelial ovarian cancer: current knowledge and future challenges. J Oncol. 2010; 2010: 568938.

11. Hirte HW. Profile of erlotinib and its potential in the treatment of advanced ovarian carcinoma. Onco Targets Ther. 2013; 6: 427-435. 
12. Reyes HD, Thiel KW, Carlson MJ, et al. Comprehensive profiling of EGFR/HER receptors for personalized treatment of gynecologic cancers. Mol Diagn Ther. 2014; 18(2): 137-151.

13. Nielsen JS, Jakobsen E, Hølund B, Bertelsen K, Jakobsen A. Prognostic significance of p53, Her-2, and EGFR overexpression in borderline and epithelial ovarian cancer. Int J Gynecol Cancer. 2004; 14(6): 1086-1096.

14. Brustmann H. Epidermal growth factor receptor expression in serous ovarian carcinoma: An immunohistochemical study with galectin-3 and cyclin D1 and outcome. Int J Gynecol Pathol. 2008; 27(3): 380-389.

15. Fujiwara S, Terai Y, Kawaguchi H, et al. GPR30 regulates the EGFR-Akt cascade and predicts lower survival in patients with ovarian cancer. J Ovarian Res. 2012; 5(1): 35.

16. van Haaften-Day C, Russell P, Boyer CM, et al. Expression of cell regulatory proteins in ovarian borderline tumors. Cancer. 1996; 77(10): 2092-2098.

17. Mehner C, Oberg AL, Goergen KM, et al. EGFR as a prognostic biomarker and therapeutic target in ovarian cancer: evaluation of patient cohort and literature review. Genes Cancer. 2017; 8(5-6): 589599.

18. Wang D, Konishi I, Koshiyama M, et al. Immunohistochemical localization of c-erbB-2 protein and epidermal growth factor receptor in normal surface epithelium, surface inclusion cysts, and common epithelial tumours of the ovary. Virchows Arch A Pathol Anat Histopathol. 1992; 421(5): 393-400.

19. Smith V, Hobbs S, Court W, Eccles S, Workman P, Kelland LR. ErbB2 overexpression in an ovarian cancer cell line confers sensitivity to the HSP90 inhibitor geldanamycin. Anticancer Res. 2002; 22(4): 1993-1999.

20. Serrano-Olvera A, Dueñas-González A, GallardoRincón D, Candelaria M, De la Garza-Salazar J. Prognostic, predictive and therapeutic implications of HER2 in invasive epithelial ovarian cancer. Cancer Treat Rev. 2006;32(3):180-190.

21. Karaferic A, Jovanovic D, Jelic S. Expression of HER2/neu, estrogen and progesterone receptors, CA 125 and CA19-9 on cancer cell membrane in patients with serous and mucinous carcinoma of the ovary. J BUON. 2009; 14(4): 635-639.

22. Nofech-Mozes S, Khalifa MA, Ismiil $\mathrm{N}$, et al. Immunophenotyping of serous carcinoma of the female genital tract. Mod Pathol. 2008; 21(9): 11471155.

23. Lee $\mathrm{CH}$, Huntsman DG, Cheang MCU, et al. Assessment of Her-1, Her-2, and Her-3 Expression and Her-2 amplification in advanced stage ovarian carcinoma. Int J Gynecol Pathol. 2005; 24(2): 147-152.

24. Lafky JM, Wilken JA, Baron AT, Maihle NJ. Clinical implications of the ErbB/epidermal growth factor (EGF) receptor family and its ligands in ovarian cancer. Biochim Biophys Acta. 2008; 1785(2): 232265.

25. Raspollini MR, Amunni G, Villanucci A, et al. HER2/neu and bcl-2 in ovarian carcinoma clinicopathologic, immunohistochemical, and molecular study in patients with shorter and longer survival. Appl Immunohistochem Mol Morphol. 2006; 14(2): 181-186.

26. Zhao D, Zhang F, Zhang W, He J, Zhao Y, Sun J. Prognostic Role of Hormone Receptors in Ovarian Cancer: A Systematic Review and Meta-Analysis. Int J Gynecol Cancer. 2013; 23(1): 25-33. 